\title{
Assessing Interviewer Performance in Approaching Reissued Initial Nonrespondents
}

\author{
Laurie Peeters $^{1}$, David De Coninck ${ }^{1}$, Celine Wuyts ${ }^{1}$, and Geert Loosveldt ${ }^{1}$
}

\begin{abstract}
Nonresponse is a repeatedly reported concern in survey research. In this article, we investigate the technique of reissuing nonrespondents to another interviewer and attempting to convert them into respondents, using data of Rounds 7 and 8 of the European Social Survey (ESS) in Belgium. The results show no marked differences between respondents interviewed by the more and the less successful interviewers, indicating that the latter are not more successful in persuading more reluctant respondents to participate. Sample units that were unsuccessfully approached in the initial phase by an interviewer with a high response rate are more difficult to convert during the reissue phase. Sample units that were unsuccessfully approached in the initial phase by an interviewer with a low response rate are easier to convert during the reissue phase.
\end{abstract}

Key words: Nonresponse; European social survey; reissuing.

\section{Introduction}

Much of the literature on nonresponse in survey research states that response rates are declining, and more efforts (more contact attempts, tailored advance and reminder letters and brochures, incentives for respondents, bonus arrangements for interviewers) should be made to stimulate participation and keep response rates up to standard (Beullens et al. 2018).

Concerns about response rates are strongly driven by the potential selectivity of nonresponse and the risk of nonresponse bias, but low response rates do not necessarily imply selectivity and bias. Empirical studies have reported that correlations between nonresponse rates and nonresponse bias are weaker than expected (Brick and Tourangeau 2017; Groves 2006; Wright 2015). In reality, additional fieldwork efforts are often focused on increasing response rates rather than reducing bias, with the underlying expectation that an increase in response rates will also result in less nonresponse bias. Reissuing initial nonrespondents requires significant resources and efforts, which might not necessarily pay off in terms of lowering nonresponse bias even if an increase in response rate is achieved. Some studies suggest that reissuing does reduce bias in the sample (Lynn and Clarke 2002), while others find no significant change (Curtin et al. 2000; Groves and Couper 2012; Stoop 2004). In recent years, in response to decreasing response rates and the increasing cost of surveys, adaptive survey design were tested. The results of the

${ }^{1}$ Catholic University of Leuven, Centre for Sociological Research, Parkstraat 45, 3000 Leuven, Belgium. Emails: laurieesteepeeters@gmail.com, david.deconinck@kuleuven.be, celine.wuyts@kuleuven.be and geert. loosveldt@kuleuven.be 
implementation of these designs suggest that putting differential fieldwork efforts into different groups of the population will lead to less biased survey results, at lower costs (Chun et al. 2018; Schouten et al. 2017). Given the need and interest for efficient allocation of the limited resources available for fieldwork, it is necessary to further investigate the impact of reissuing on nonresponse rates and bias. The current article adds to the literature in this field by investigating the effectiveness of reissuing as a common fieldwork practice. While the general concept of reissuing is well documented, the organization of the practice and its effect on the composition of the respondent group has not been documented in great detail. We specifically explore whether the reissuing procedure can be optimized by using information about the response rates achieved by interviewers during the initial fieldwork phase (i.e., before the start of reissuing). In addition, we also investigate whether the risk of nonresponse bias is influenced by the reissuing procedure.

\section{The Reissuing Procedure and the Role of the Interviewer}

The term "reissuing" is used to describe the process of reattempting to contact sample units that initially did not participate in a survey. Although one can question whether high response rates should be a primary objective in survey practice (Beullens and Loosveldt 2012), the main objective of a reissue procedure is to increase these rates. Instead of accepting the targeted sample unit's initial response outcome, the survey organization may choose to make further attempts to convert these cases into interviews. These additional attempts can be made by the same interviewer, or a different one (Burton et al. 2006; Tarnai and Moore 2008). Reissuing can cover any or all sources of nonresponse (refusal, noncontact, and others, possibly including ineligibility). It can thus be considered a generalization of refusal conversion, which is aimed solely at converting those cases that refused to participate during the initial fieldwork phase.

Reissuing initial nonrespondents presumes that target respondents do not thoroughly deliberate the decision to participate or not, and a refusal decision may therefore be overturned (Groves and Couper 2012). A different interviewer applying a different contact schedule or a different doorstep approach may be able to convert an initial nonresponse outcome into a completed interview.

Interviewers play a crucial role in the success of both the initial fieldwork phase and any reissue activities. Previous research into interviewer effects on nonresponse clearly demonstrates significant differences between interviewers in terms of contact and cooperation rates (Blom et al. 2011; Durrant et al. 2010; O'Muircheartaigh and Campanelli 1999; Pickery and Loosveldt 2002), indicating that some interviewers are more successful than others in contacting targeted sample units and persuading them to cooperate. These interviewer effects also create differential nonresponse error across interviewers (West and Olson 2010), so it is doubtful that nonresponse within interviewers is completely random. This brings us to the question of which interviewers might be more successful at getting reissued nonrespondents to participate, and thus should preferably be selected for reissue activities?

Interviewers may be selected for reissuing on the basis of their gender, age, or ethnic background in order to maximize the likelihood of converting nonrespondents with similar characteristics (Gideon 2012). Typically, however, the better trained, more 
experienced interviewers or those who achieved a high response rate in the first phase of the fieldwork or in previous surveys are selected for the reissue phase. Previous studies of the effectiveness of reissue procedures suggest that when highly performing interviewers from the initial phase are deployed in reissue activities, conversion rates are significantly affected in a positive way (Beullens et al. 2009; Stoop et al. 2014). These studies have not examined whether respondents interviewed by "high-performance" interviewers are different from respondents interviewed by their less successful colleagues in terms of socio-demographic or socio-political characteristics. One can assume that interviewers with lower response rates follow the line of least resistance and focus on the "low hanging fruit" (Beullens et al. 2009), mostly interviewing people with a higher response propensity (e.g., those spending more time at home or having greater interest in the topic of the survey). On the other hand, interviewers with higher response rates may be able to track, contact, and convince more reluctant targets and those that are harder to reach.

In this study, we investigate the process of reissuing and its effect on the survey response rate and the composition of the net sample in Round 7 and Round 8 of the European Social Survey (ESS) in Belgium. We do this by identifying the interviewers engaged in the initial fieldwork efforts and reissue phase, comparing their response rates in each phase of the fieldwork, and investigating socio-demographic and socio-political differences between the groups of respondents that are interviewed in each part of the fieldwork. We formulate the following hypotheses:

Hypothesis 1: Compared to interviewers who achieve low response rates in the initial phase, interviewers who achieve high response rates in the initial phase interview respondents with a different profile.

Hypothesis 2: Interviewers who achieve high response rates in the initial phase are also more successful, in terms of achieved response rates, in the reissue phase.

Hypothesis 3A: Nonrespondents approached in the initial phase by an interviewer with a high response rate are more difficult to convert during the reissue procedure.

Hypothesis 3B: Nonrespondents approached in the initial phase by an interviewer with a low response rate might have completed an interview during the first phase if they had been approached by an interviewer with a high response rate.

\section{Data}

Data from Round 7 and Round 8 of the European Social Survey (ESS) in Belgium are used to test the hypotheses. The same organization was responsible for fieldwork in both rounds, and there are several interviewers who were deployed in both rounds. Fieldwork for Round 7 started on 15 September 2014 and ended approximately 20 weeks later, on 1 February 2015. For Round 8, the fieldwork also took about 20 weeks (14 September 2016 until 31 January 2017). The total number of issued sample units for each round is 3,204 (Barbier et al. 2016; Wuyts et al. 2018). See the documentation reports of ESS7 (European Social Survey 2015) and ESS8 (European Social Survey 2017) for more information on the survey designs. Both rounds are separately analyzed so that we can compare and validate the results. 
The reissue procedure is delineated by a new interviewer having been assigned to a case after the first interviewer had completed all contact attempts in the initial contact phase without obtaining an interview (Gideon 2012). In the current article, all types of initial nonresponse (noncontacts, refusals, other nonresponse, and ineligibility) are included. It was specified in the contract with the fieldwork organization that these sample units would be re-allocated to a different interviewer, who would apply the contact procedure all over again, using the information available from previous contacts. The interviewers used in the refusal conversion procedure would be selected based on their response performance in the running ESS project and previous projects. Within the constraints of these contract specifications, the actual selection of the interviewers and the sample units for reissuing was at the discretion of the survey agency.

For Round 7, the 151 interviewers that began fieldwork completed a total of 1,506 interviews out of the 3,204 potential respondents (initial response rate $=47 \%$ ). Out of the 1,698 individuals that did not complete an interview, 1,040 (61\%) were reissued and reattempted face-to-face by a new interviewer. Round 8 started off with 139 interviewers who completed 1,475 interviews in the initial phase (initial response rate $=46 \%$ ). Half of the initial nonrespondents (861 cases) were reissued in the traditional, face-to-face mode. Some 389 additional cases were assigned to the survey agency's call center and assigned to a face-to-face interviewer only after agreeing to participate. In order to ensure comparability, this latter part of the reissuing procedure in Round 8 is not included in the analysis of the reissue phase. Reissuing was finalized at these numbers, because an acceptable response rate was achieved and to increase it further would have required additional means that were not available.

Most cases selected for reissues in either round had refused to participate in the initial phase. Initial noncontacts also make up a large proportion of reissues, whilst the other two sample unit groups (initial "others" and ineligibles) are much smaller (see Table 8 in Appendix, Section 7).

The distribution of response outcomes after the initial and the reissue phase for Rounds 7 and 8 indicates that a total of 263 additional interviews in Round 7 (25\% of the reissued cases), and 225 interviews in Round 8 ( $26 \%$ of the reissued cases) were conducted during the reissue phase, most of which were initial refusals. The response rate for reissues was thus about half as large as the response rate of the initial phase (25\% and $47 \%$ in Round 7, 26\% and $46 \%$ in Round 8). At the end of fieldwork activities in Round 7, 1769 interviews had been obtained (response rate $=57 \%$ ) and at the end of Round 8, 1766 interviews were realized (response rate $=56.8 \%$ ). For more information on the response outcomes after the initial and reissue phase, see Table 9 and Table 10 in Appendix. For both rounds, the objective of attaining an acceptable response rate was realized by virtue of the extensive reissuing of nonrespondents (adding 10-11 percentage points). In the next section, we investigate which interviewers were selected and were most successful in the reissue procedure.

\section{Interviewers' Response Rates and the Impact on Respondent Profiles During the Initial Fieldwork Phase}

In Round 7, the average response rate of an interviewer in the initial phase was $48.0 \%$ $(\mathrm{S} D=19.3)$. Only 10 interviewers $(6.6 \%)$ had a response rate below or equal to $20 \%$, 
Table 1. Interviewer variance and the ICCs for the multilevel logistic null models with the dependent variable response/nonresponse in the initial phase and the interviewer at the second level.

\begin{tabular}{lll}
\hline & ESS7 & ESS8 \\
\hline Interviewer variance & $0.3529 *$ & $0.3547^{*}$ \\
ICC & 0.0969 & 0.0973 \\
\hline
\end{tabular}

$* \mathrm{p}<0.001$

40 interviewers $(26.5 \%)$ had a rate between $20 \%$ and $40 \%, 62$ interviewers $(41.1 \%)$ had a rate between $40 \%$ and $60 \%$, and 39 interviewers $(25.8 \%$ ) achieved a response rate more than $60 \%$. In Round 8, the average response rate of an interviewer was $45.3 \%$ ( $S D=17.4$ ). Only 11 interviewers $(7.9 \%$ ) had a response rate below or equal to $20 \%$, 40 interviewers (28.8\%) had a rate between $20 \%$ and $40 \%, 57$ interviewers $(41.0 \%)$ had a rate between $40 \%$ and $60 \%$, and 31 interviewers $(22.3 \%$ ) achieved a response rate over $60 \%$.

In order to formally test these differences, a multilevel logistic regression model with sample units nested within interviewers was estimated to predict the probability of obtaining an interview in the initial phase for both rounds. The interviewer variance for the null model, including only the random intercept effect and no explanatory variables, and the intraclass correlation coefficients (ICCs) are presented in Table 1. The ICCs for the two null models suggest that $9.7 \%$ of the variability in the obtained participation of sample units in the initial phase is explained by between-interviewer differences. These estimates provide evidence that some interviewers are more successful than others.

\subsection{Hypothesis 1: Compared to interviewers who achieve low response rates in the initial phase, interviewers who achieve high response rates in the initial phase interview respondents with a different profile}

The question is whether interviewers with higher response rates interview respondents with different socio-demographic or socio-political profiles than interviewers with lower response rates. To answer this question and test the first hypothesis, interviewers were divided into two groups: those with an individual response rate in the initial phase higher than the overall response rate of the survey and those with an individual response rate in the initial phase lower than the overall response rate. The overall response rates for Rounds 7 and 8 are $57.0 \%$ and $56.8 \%$, respectively. These cut-offs are also used in the following sections to differentiate interviewers with low response rates and those with high response rates. We expect interviewers with a low response rate to mainly interview respondents who are more easily persuaded to participate, whereas interviewers with a high response rate in the initial phase are expected to be better at contacting and persuading more reluctant or hard-to-reach-respondents.

In Table 2, we compare respondent profiles of interviewers with a low response rate to those from interviewers with a high response rate. There are no significant differences in respondent profiles between the two groups of interviewers in either round regarding respondents' age, gender, work situation, and the presence of children in the household. The expectation that interviewers with high response rates would interview more hard-toreach-respondents due to being employed or having children in the household is thus not 


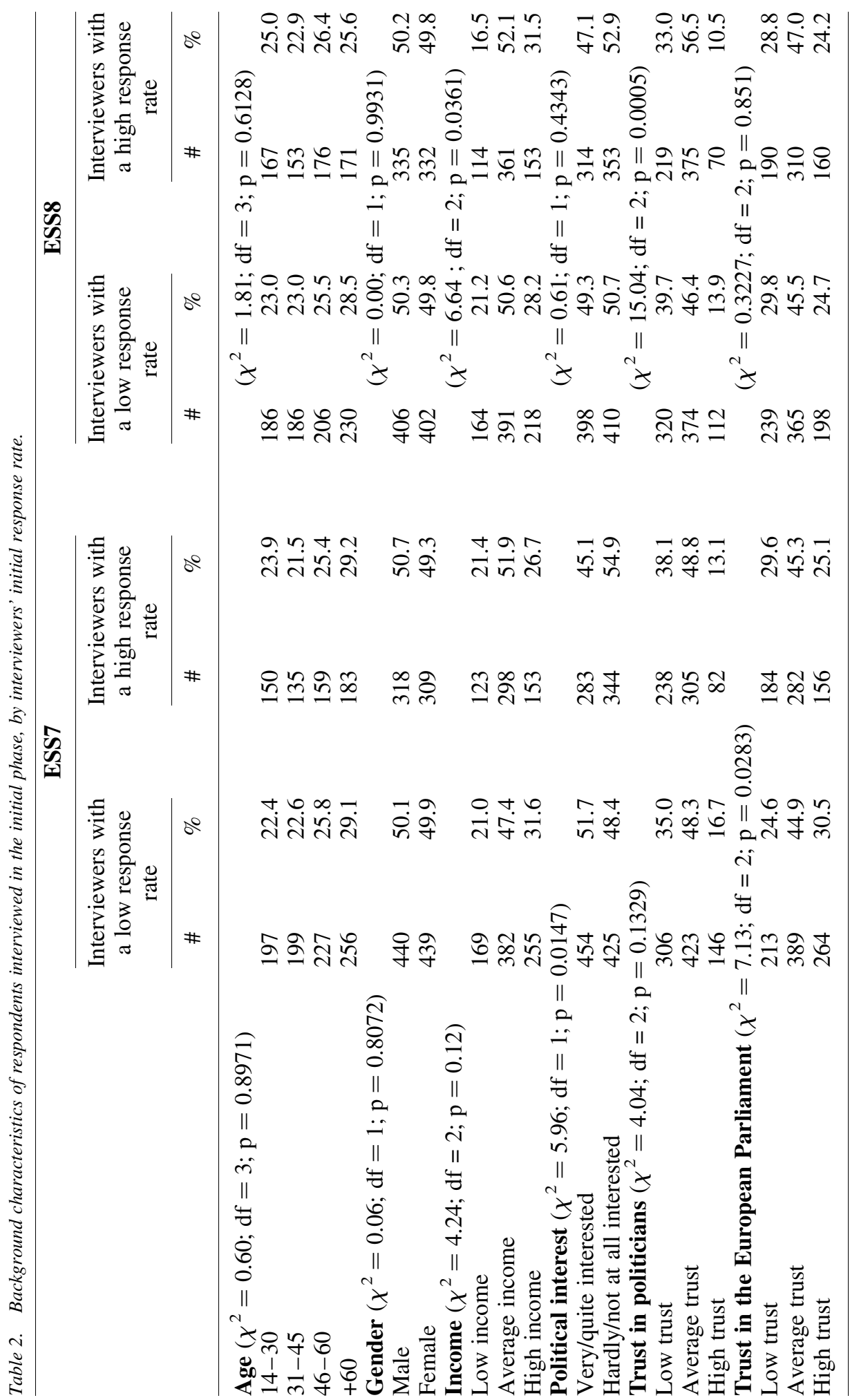




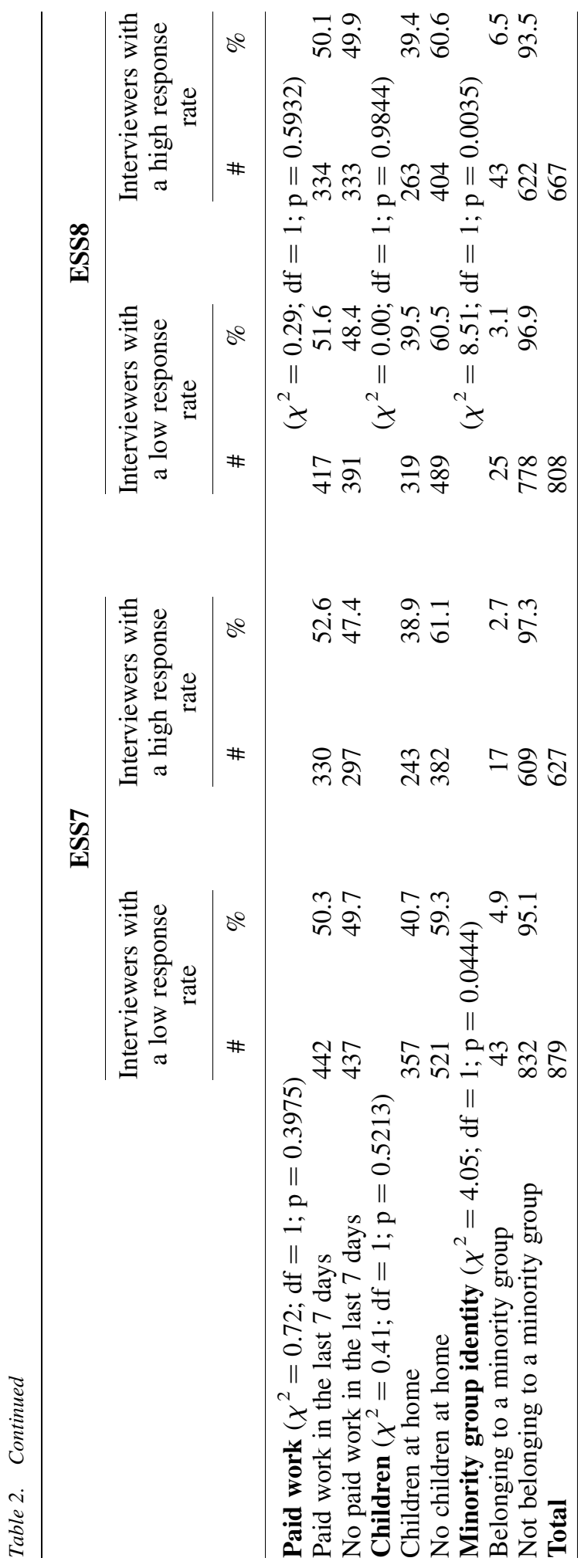


confirmed. In Round 8, there is a significant difference concerning respondents' income: interviewers with a high initial response rate are more likely to interview respondents with a higher income than interviewers with a low initial response rate. It must be noted that there is some item nonresponse for the income variable, but this is never larger than $10 \%$. Although the results are mixed, there are some indications that interviewers with a high response rate interview a greater number of respondents with less interest and trust in politics. In line with expectations, there is a tendency for interviewers with a high response rate to be more successful in interviewing respondents that are hardly or not at all interested in politics (only significant in Round 7), respondents with average confidence in politicians (only for Round 8) and respondents with little confidence in the European Parliament (only for Round 7). In Round 8, interviewers with a high response rate also interviewed more respondents belonging to a minority group, but the opposite pattern is observed for Round 7.

When analysing respondents' characteristics for interviewers with a low response rate as opposed to interviewers with a high response rate, no strong evidence is found to support the hypothesis that interviewers who achieve high response rates in the initial phase also interview respondents with a different socio-demographic or socio-political profile compared to interviewers with low response rates in the initial phase.

\section{The Impact of the Interviewers on the Success of the Reissue Phase}

After the initial phase, initial nonrespondents were assigned to another interviewer to reattempt contact and persuade them to participate. In Table 3, respondent characteristics of both phases are compared. In both rounds, significantly more respondents with a low interest in politics were interviewed in the reissue phase than in the initial phase. Other significant differences between the initial and the reissue phase arise, but are not consistent across the two rounds. In Round 7, a smaller percentage of respondents with a high level of trust in the European Parliament and a higher percentage of respondents from a minority group were interviewed in the reissue phase than in the initial phase. In Round 8, a higher percentage of young people, people with a lower income, and people with an average trust in politicians were interviewed. Although the specifics are inconsistent, these results suggest that different types of respondents are interviewed in the reissue phase.

Typically, only a subset of the interviewers working in the initial phase of fieldwork are selected to participate in the reissue phase. Of the 46 interviewers with a high response rate in the initial phase of Round 7, 27 (58.7\%) were engaged in the reissue phase. Most of the interviewers in the reissue phase of Round 7 (53 out of 62 or $85 \%$ ) had a respectable to very good response rate (over 40\%) in the initial contact phase. In Round 8, a similar pattern emerges: of the 39 interviewers with a high response rate in the initial phase, 27 $(69.2 \%)$ were employed in the reissue phase, and $63 \%$ of the interviewers used for reissuing had an initial response rate higher than $40 \%$. These results are in line with expectations based on previous literature and experience with survey agencies that suggest that the selection of the interviewers is driven by their recorded performance (in addition to availability and travel costs), and that response rates that were previously 


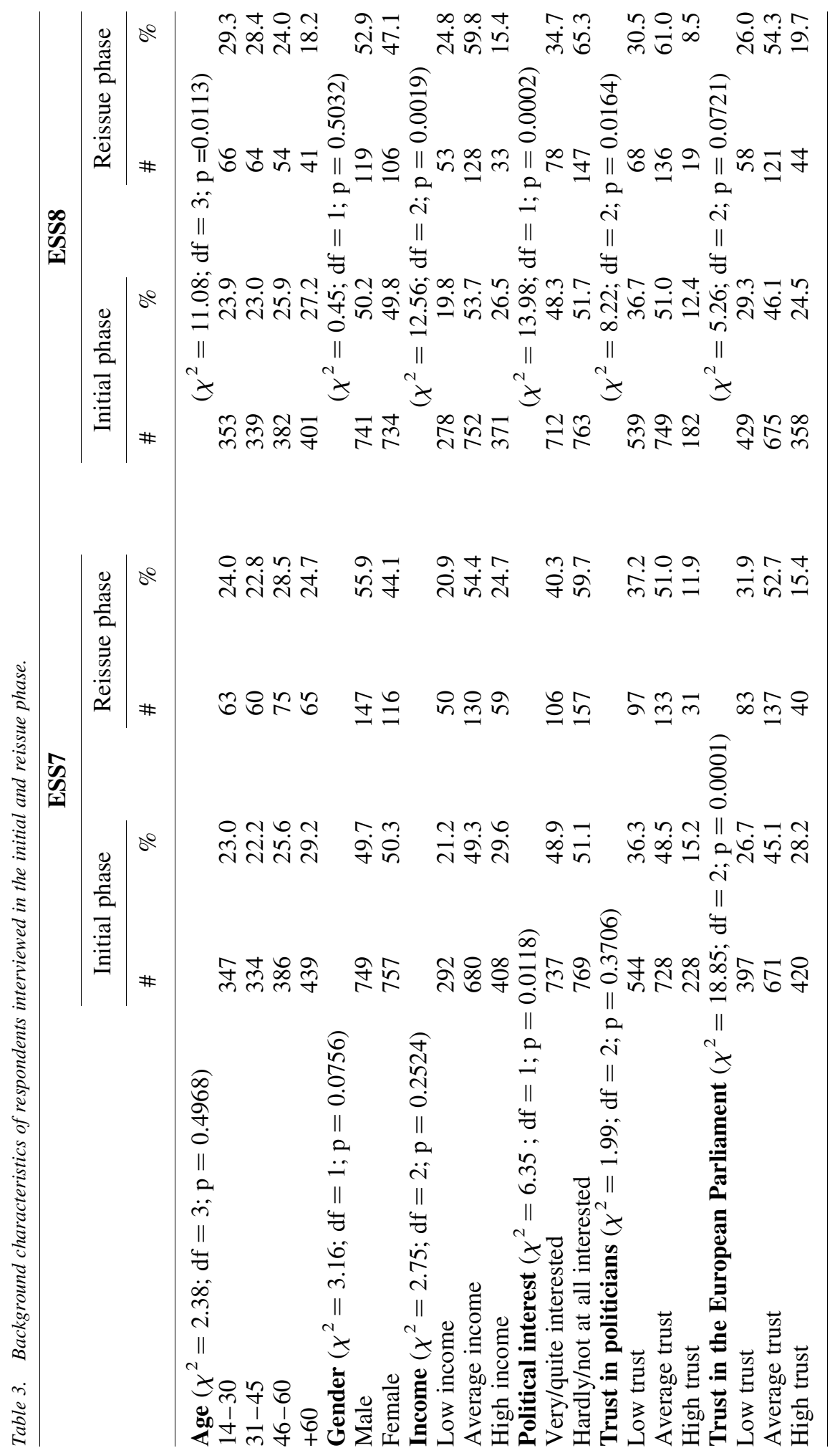




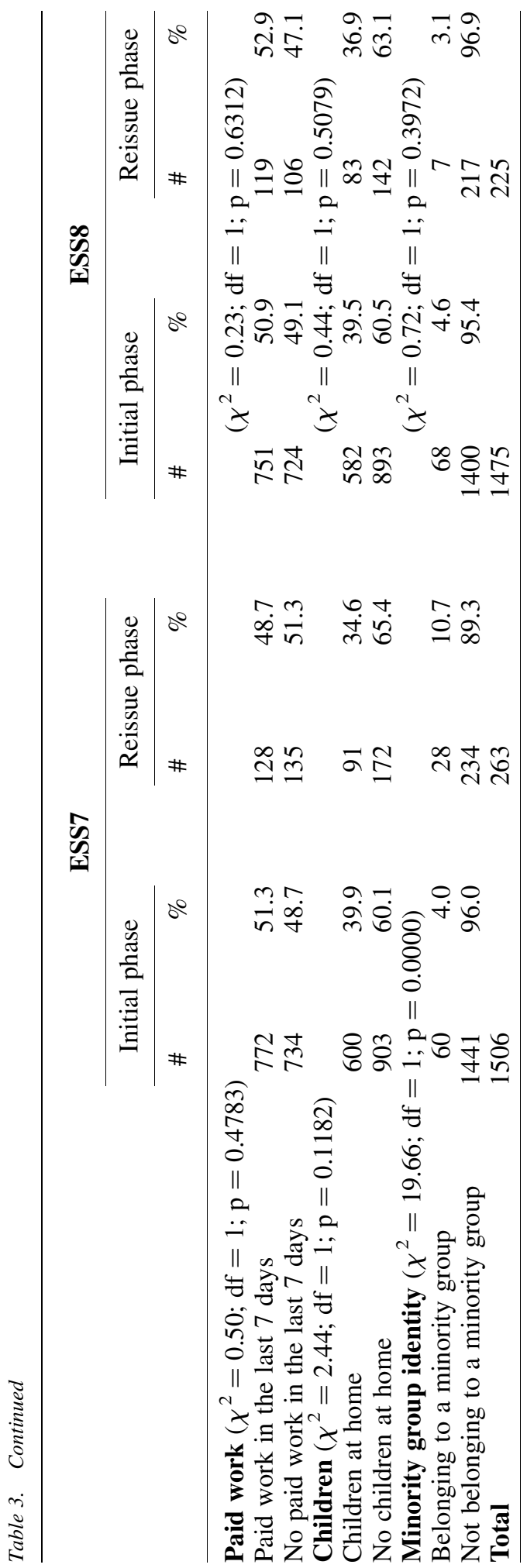


achieved (e.g., in an earlier phase of fieldwork) may serve as a useful criterion in the selection.

Three additional hypotheses about the impact of the interviewers' response rates in the initial phase on the results in the reissue phase are tested.

\subsection{Hypothesis 2: Interviewers who achieve high response rates in the initial phase are also more successful, in terms of achieved response rates, in the reissue phase}

Table 4 presents the mean response rates achieved in the reissue phase for interviewers with a low initial response rate and those with a high one. These results support the hypothesis: interviewers with a high response rate in the initial phase achieve a higher response rate in the reissue phase, but this difference at the interviewer level is not statistically significant. In the reissue phase, the interviewers fail to realize response rates that are comparable to those in the initial phase. This indicates that sample units in the reissue phase are more difficult to convert. It is clear that the sample units that were still not interviewed after the reissue phase may be considered as "high hanging fruit", but the characteristics of this remaining group of nonrespondents cannot be observed.

To elaborate and refine the results at the sample unit level, a multilevel logistic regression model with a random intercept is estimated to predict the probability of an interview in the reissue phase based on the interviewers' response rates in the initial phase. For both rounds, the results indicate that the interviewers' initial response rate is indeed a positive predictor of target sample units' participation in the reissue phase. Interviewers who achieved high response rates in the initial phase had a greater likelihood of obtaining participation from the sample units assigned to them in the reissue phase. This is in line with the results in Table 4.

\subsection{Hypothesis 3A: Nonrespondents approached in the initial phase by an interviewer} with a high response rate are more difficult to convert during the reissue procedure

Although strong and systematic differences between the respondent groups interviewed in the initial phase by interviewers with a high response rate and by interviewers with a low response rate could not be identified, one assumes that sample units unsuccessfully approached by interviewers with a high response rate in the initial phase will be more difficult to convert during the reissue phase than those approached by interviewers with a low response rate in the initial phase.

In Round 7, there is a higher percentage of reissued sample units participating in the survey if they were approached by an interviewer with a lower response rate in the initial

Table 4. Mean response rates in the reissue phase for interviewers with low and high initial response rates.

\begin{tabular}{lcc}
\hline & $\begin{array}{c}\text { Low initial response } \\
\text { rate }\end{array}$ & $\begin{array}{c}\text { High initial response } \\
\text { rate }\end{array}$ \\
\hline $\begin{array}{l}\mathrm{ESS7} \\
\quad t=-1.58 ; d f=54.42 ; p=0.06)\end{array}$ & $19.35 \%$ & $28.08 \%$ \\
$\mathrm{ESS} 8$ & $26.16 \%$ & $29.13 \%$ \\
$\quad(t=-0.68 ; d f=171.74 ; p=0.25)$ & & \\
\hline
\end{tabular}


phase $(26.1 \%)$ than if they were approached by an interviewer with a higher response rate in the initial phase $(21.4 \%)$, but the difference (4.7 percentage points) is not statistically significant $\left(X^{2}=1.42 ; d f=1 ; p=0.2313\right)$. In Round 8 , the opposite pattern is observed, as there appears to be a higher but not statistically significant percentage of participating reissued sample units if they were approached by an interviewer with a higher initial response rate $(27.2 \%)$ rather than by an interviewer with a lower initial response rate (25.9\%) $\left(X^{2}=0.04 ; d f=1 ; p=0.8382\right)$. However, when using the continuous response rates in a multilevel logistic model, taking into consideration the random effect of the interviewers, the initial interviewers' response rate becomes statistically significant (see Table 5). This suggests that there is an effect of the initial interviewers' response rate based on the continuous response rates instead of on the binary separation of low and high response rates. This estimate is negative, suggesting that nonrespondents who were approached by an interviewer with a higher response rate are more difficult to convert in the reissue phase, as suggested in the hypothesis.

\subsection{Hypothesis 3B: Nonrespondents approached in the initial phase by an interviewer with a low response rate might have completed an interview during the first phase if they had been approached by an interviewer with a high response rate}

Based on hypothesis $3 \mathrm{~B}$, we expect that there is an interaction effect on the conversion rate between the response rate of the interviewer in the first phase (interviewer 1) and the initial response rate of the interviewer in the reissue phase (interviewer 2). Such an interaction effect would imply that nonrespondent sample units approached in the initial phase by an interviewer with a low response rate might very well have completed an interview during the first phase if they had been approached by an interviewer with a high response rate.

The results in Table 5 and Table 6 show that in both rounds, interviewers with high initial response rates successfully converted about one third of the reissued cases when these had been approached in the initial phase by an interviewer with a low response rate (Round 7: 35.7\%; Round 8: 32.8\%), which is significantly more than interviewers with low initial response rates (Round 7: 17.9\% $(t=-5.9 ; d f=764.1 ; p<0.01$ ); Round 8: $17.7 \%(t=-2.3 ; d f=288.8 ; p=0.03)$. In Round 7 , the difference in successfully interviewed reissued cases between the group of interviewers with high initial response rates and those with low initial response rates is larger for the sample

Table 5. Distribution of the conversion rate by response rate of interviewer 1 and interviewer 2 for Round 7 .

\begin{tabular}{|c|c|c|c|c|c|c|c|}
\hline \multirow{3}{*}{$\begin{array}{l}\text { Response rate } \\
\text { interviewer } 1\end{array}$} & \multirow{3}{*}{$\begin{array}{c}\text { Response rate } \\
\text { interviewer } 2\end{array}$} & \multicolumn{4}{|c|}{ Conversion } & & \\
\hline & & \multicolumn{2}{|c|}{ No } & \multicolumn{2}{|c|}{ Yes } & \multicolumn{2}{|c|}{ Total } \\
\hline & & $\#$ & $\%$ & \# & $\%$ & \# & $\%$ \\
\hline \multirow[t]{2}{*}{ Low } & Low & 376 & 82.1 & 82 & 17.9 & 458 & 53.3 \\
\hline & High & 258 & 64.3 & 143 & 35.7 & 401 & 46.7 \\
\hline \multirow[t]{2}{*}{ High } & Low & 79 & 82.3 & 17 & 17.7 & 96 & 55.8 \\
\hline & High & 56 & 73.7 & 20 & 26.3 & 76 & 44.2 \\
\hline
\end{tabular}


Table 6. Distribution of the conversion rate by response rate of interviewer 1 and interviewer 2 for Round 8 .

\begin{tabular}{|c|c|c|c|c|c|c|c|}
\hline \multirow{3}{*}{$\begin{array}{l}\text { Response rate } \\
\text { interviewer } 1\end{array}$} & \multirow{3}{*}{$\begin{array}{l}\text { Response rate } \\
\text { interviewer } 2\end{array}$} & \multicolumn{4}{|c|}{ Conversion } & & \\
\hline & & \multicolumn{2}{|c|}{ No } & \multicolumn{2}{|c|}{ Yes } & \multicolumn{2}{|c|}{ Total } \\
\hline & & $\#$ & $\%$ & \# & $\%$ & $\#$ & $\%$ \\
\hline \multirow[t]{2}{*}{ Low } & Low & 390 & 76.2 & 122 & 23.8 & 512 & 74.0 \\
\hline & High & 121 & 67.2 & 59 & 32.8 & 180 & 26.0 \\
\hline \multirow[t]{2}{*}{ High } & Low & 50 & 78.1 & 14 & 21.9 & 64 & 50.4 \\
\hline & High & 40 & 63.5 & 23 & 36.5 & 63 & 49.6 \\
\hline
\end{tabular}

units interviewed in the initial phase by an interviewer with a low response rate. In Round 8 , the expected difference is present for the group of sample units that was approached in the initial phase by an interviewer with a low response rate. In the group with an interviewer with a high initial response rate, the difference in conversion rates is even larger.

However, the interaction between the two initial response rates is not statistically significant; both either tested with the binary or with the continuous response rate variables. This implies that there is no difference in the second interviewer's conversion rate of the sample units based on the initial interviewer's response rate. However, when only including the main effects, the initial response rates of the interviewers become significant (Table 7). These results apply to both Round 7 and Round 8. As expected, there is a negative main effect of the first interviewer's initial response rate on conversion in the reissue phase: the higher the response rate of the interviewer in the initial phase, the lower the conversion rate in the reissue phase. There is, also as expected, a positive main effect of the second interviewer's initial response rate: the higher the second interviewer's initial response rate, the higher the conversion rate. These coefficients are small due to the fact that a change of one percentage point in the response rate is small, but larger percentage differences will have a greater impact on the conversion of the sample unit.

The results of the logistic regression analysis (Table 7) also indicate that in both rounds, conversion rates are significantly higher for soft refusals (refusals for which the initial interviewer indicates that the sample unit might still be persuaded to participate) and the "other" nonresponse category. In Round 7, the conversion rate is significantly lower for female respondents, as well as for the 31-45 and the over 60 age groups when compared with the youngest category.

\section{Conclusion and Discussion}

Our results, based on data from two rounds of the European Social Survey in Belgium, suggest that in both the initial and the reissue phase, some interviewers are more successful than others in contacting sample units and convincing them to participate in the survey. Nevertheless, there is no strong evidence that interviewers with a higher response rate interview more reluctant sample units in the initial phase than interviewers with a lower response rate. 
Table 7. Logistic regression models predicting conversion in the reissue phase (odds ratios).

\begin{tabular}{|c|c|c|}
\hline & \multicolumn{2}{|c|}{ Conversion of reissues } \\
\hline & ESS7 & ESS8 \\
\hline Constant & $0.13 * * *$ & $0.23 * * *$ \\
\hline \multicolumn{3}{|l|}{ Initial contact outcome } \\
\hline Soft refusal & $2.04 * *$ & $2.16^{*}$ \\
\hline Noncontact & 1.32 & 1.25 \\
\hline Other & $3.31 * *$ & $2.58 *$ \\
\hline \multicolumn{3}{|l|}{ Age of case } \\
\hline $31-45$ years old & $0.55 * *$ & 0.88 \\
\hline $46-60$ years old & 0.70 & 0.90 \\
\hline+60 years old & $0.64 *$ & 0.93 \\
\hline \multicolumn{3}{|l|}{ Gender of case } \\
\hline Female & $0.70 *$ & 0.89 \\
\hline \multicolumn{3}{|l|}{ Initial response rates of interviewers } \\
\hline Initial response rates interviewer 1 & $0.98 * * *$ & $0.98 * * *$ \\
\hline Initial response rates interviewer 2 & $1.03 * * *$ & $1.02 * * *$ \\
\hline
\end{tabular}

Interviewers with a higher response rate in the initial phase are also more successful in the reissue phase, and there is evidence that nonrespondents approached by an interviewer with a high response rate in the initial phase are more difficult to convert during the reissue phase.

Another question is whether the reissue phase is worth the investment. The most important effect is increasing the response rate, with an increase of the statistical power as a result. The higher response rate may also increase people's confidence in the results. It can be noted that the response rate can also be increased by using higher-skilled and successful interviewers from the beginning and eliminating interviewers with low response rates. For future ESS rounds, we recommend looking at the skills of interviewers based on participation in the fieldwork of previous ESS rounds and similar projects if possible and use this information to select the best possible interviewers for both the initial and the reissue phase. The impact of reissuing on the risk of nonresponse bias seems limited, with only marginal changes found in the socio-demographic and socio-political composition of the respondent groups in the initial and reissue phase. There are some indications that different types of respondents are interviewed during the reissue phase, but the differences are small and inconsistent for the two rounds examined. As the effect of re-issuing on the sample composition is not particularly large, the impact on nonresponse bias is likely not large either.

It can be said that the sample units that have still not been interviewed after the reissue phase are very difficult to contact and persuade to take part in an interview. However, based on the available content variables, it was not possible to further characterize this group of ultimate nonrespondents.

The information about the implementation of the refusal conversion procedure during the fieldwork is limited. According to the contract specifications with the fieldwork 
organization, we can assume that the fieldwork organization selected the best performing interviewers that were available in an area. For future research, more transparency of the survey agency regarding the selection of the interviewers (e.g., their initial response rate, years of experience, socio-demographic characteristics, type of region - urban or rural in which they work) and sample units (e.g., initial response outcome, sociodemographic characteristics) for the reissue phase would be desirable. Especially more information about the selection of the sampling units that are being reissued can contribute to a more thorough evaluation of the procedure. We can assume that the fieldwork organization applies a pragmatic approach. It is important to know which considerations (e.g., geographical proximity of sample units, availability of interviewers with, type of nonresponse) play a role in this process, and how different considerations are weighed against each other.

Another limitation of this study lies in the differential nature of the contact procedure in the reissue phase: in Round 8, 389 reissued cases were assigned to call center reissuing and went on to a face-to-face interview only after agreeing to participate, while all reissues in Round 7 were done via the face-to-face mode. Because of this discrepancy, these 389 cases were only included in the analysis of the initial nonresponse. This means that part of the picture on reissue activities in Round 8 was not taken into account. However, it is possible that reissued cases in the ESS8 may be more difficult sample units than in ESS7. Future studies that evaluate the effectiveness of reissues in increasing response rates and reducing bias (especially in repeated surveys such as the European Social Survey) should consider that survey organizations try to optimize their scarce means every step of the way to maximize the response rate and reduce bias. Research into the impact of the contact procedure (e.g., telephone versus face-to-face) during the reissuing phase may contribute to this.

\section{Appendix}

Table 8. Distribution of initial response outcomes of cases selected for reissuing.

\begin{tabular}{lcccccr}
\hline & & Noncontact & Refusal & Other & Ineligible & Total \\
\hline Reissued cases ESS7 & $\#$ & 263 & 740 & 33 & 4 & 1040 \\
& $\%$ & 25.3 & 71.2 & 3.2 & 0.4 & 100 \\
\hline \multirow{2}{*}{ Reissued cases ESS8 } & $\#$ & 298 & 530 & 29 & 4 & 861 \\
& $\%$ & 34.6 & 61.6 & 3.4 & 0.5 & 100 \\
\hline
\end{tabular}




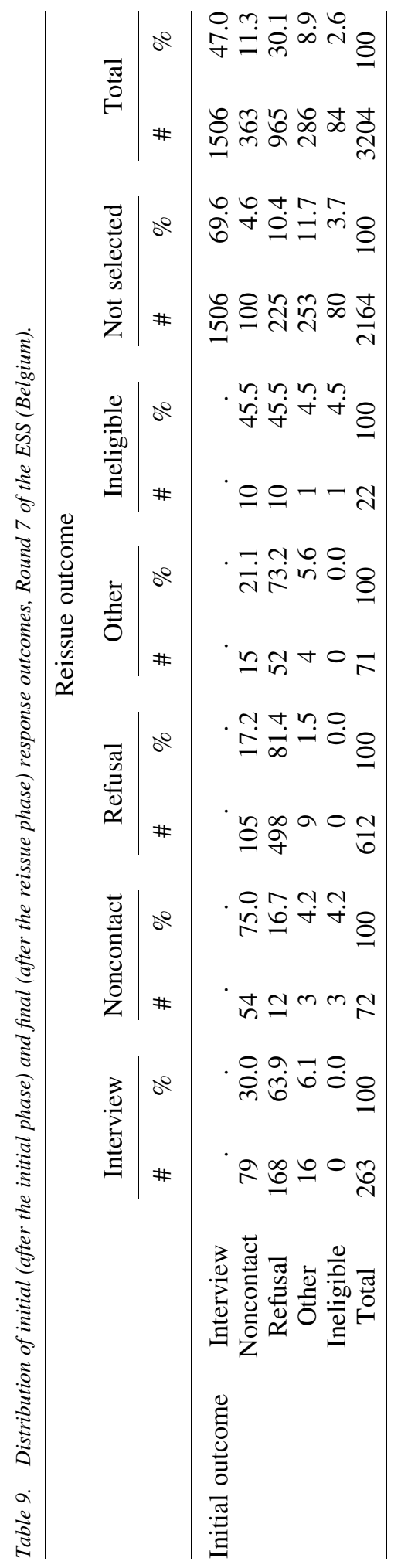




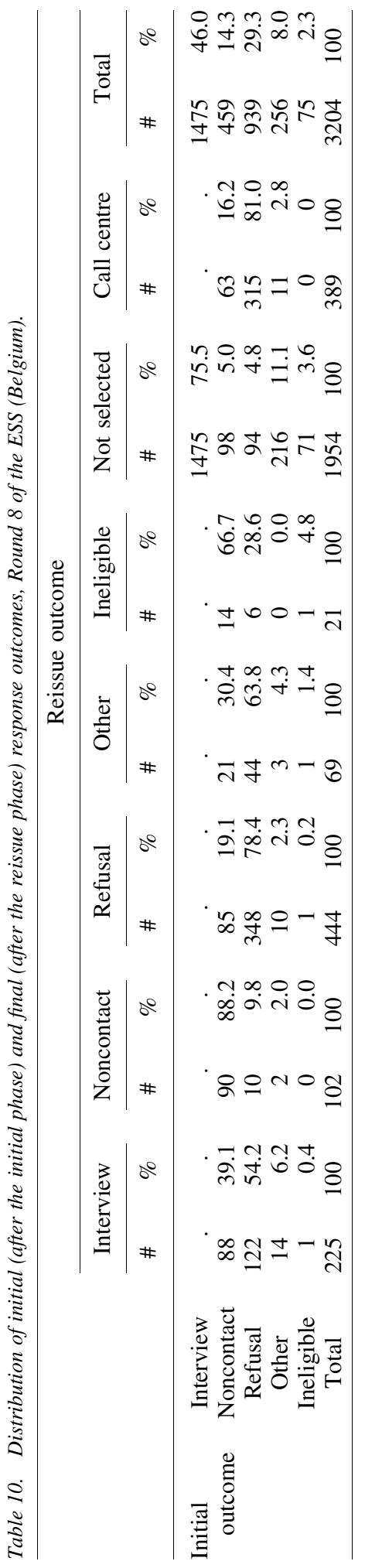




\section{References}

Barbier, S., C. Wuyts, P. Italiano, and G. Loosveldt. 2016. European Social Survey Round 7 Belgium: Process evaluation for the data collection. Leuven: KU Leuven.

Beullens, K., J. Billiet, and G. Loosveldt. 2009. Selection strategies for refusal conversion of four countries in the European Social Survey, 3rd round. Leuven: KU Leuven.

Beullens, K. and G. Loosveldt. 2012. "Should high response rates really be a primary objective?" Survey Practice 5(3): 1-5. DOI: https://doi.org/10.29115/SP-2012-0019.

Beullens, K., G. Loosveldt, C. Vandenplas C., and I. Stoop. 2018. "Response rates in the European Social Survey: Increasing, decreasing, or a matter of fieldwork efforts? Survey methods: Insights from the field." Available at: https://surveyinsights.org/?p = 9673 (accessed May 2020).

Blom, A., E. de Leeuw, and J. Hox. 2011. "Interviewer effects on nonresponse in the European Social Survey.” Journal of Official Statistics 27(2): 359-377. Available at: https://www.scb.se/contentassets/ca21 efb41fee47d293bbee5bf7be7fb3/interviewereffects-on-nonresponse-in-the-european-social-survey.pdf (accessed May 2020).

Brick, J.M. and R. Tourangeau. 2017. "Responsive survey designs for reducing nonresponse bias.” Journal of Official Statistics 33(3): 735-752. DOI: https://doi.org/ 10.1515/jos-2017-0034.

Burton, J., H. Laurie, and P. Lynn. 2006. "The long-term effectiveness of refusal conversion procedures on longitudinal surveys." Journal of the Royal Statistical Society. Series A (Statistics in Society) 169(3): 459-478. DOI: https://doi.org/10.1111/ j.1467-985X.2006.00415.x.

Chun, A.Y., S.G. Heeringa, and B. Schouten. 2018. "Responsive and adaptive design for survey optimization.” Journal of Official Statistics 34(3): 581-597. DOI: https:// doi.org/10.2478/jos-2018-0028.

Curtin, R., S. Presser, and E. Singer. 2000. "The effects of response rate changes on the index of consumer sentiment." Public Opinion Quarterly 64(4): 413-428. DOI: https:// doi.org/10.1086/318638.

Durrant, G., R. Groves, L. Staetsky, and F. Steele. 2010. "Effects of interviewer attitudes and behaviors on refusal in household surveys". Public Opinion Quarterly 74(1): 1-36. DOI: https://doi.org/10.1093/poq/nfp098.

European Social Survey. 2015. ESS Round 7 (2014/2015) Technical Report. London: ESS ERIC.

European Social Survey. 2017. ESS Round 8 (2016/2017) Technical Report. London: ESS ERIC.

Gideon, L. 2012. Handbook of survey methodology for the social sciences. New York: Springer.

Groves, R. 2006. "Nonresponse rates and nonresponse bias in household surveys." Public Opinion Quarterly 70(5): 646-675. DOI: https://doi.org/10.1093/poq/nfl033.

Groves, R.M. and M.P. Couper. 2012. Nonresponse in household interview surveys. Hoboken, New Jersey: John Wiley \& Sons.

Lynn, P. and P. Clarke. 2002. "Separating refusal bias and non-contact bias: Evidence from UK national surveys." Journal of the Royal Statistical Society 51(3): 319-333. DOI: https://doi.org/10.1111/1467-9884.00321. 
O’Muircheartaigh, C. and P. Campanelli. 1999. "A multilevel exploration of the role of interviewers in survey non-response." Journal of the Royal Statistical Society: Series A (Statistics in Society) 162(3): 437-446. DOI: https://doi.org/10.1111/1467985X.00147.

Pickery, J. and G. Loosveldt. 2002. "A multilevel multinomial analysis of interviewer effects on various components of unit nonresponse." Quality and Quantity 36(4): 427-437. DOI: https://doi.org/10.1023/A:1020905911108.

Schouten, B., A. Peytchev, and J. Wagner. 2017. “Adaptive Survey Design.” Series on Statistics Handbooks, Chapman and Hall/CRC.

Stoop, I. 2004. "Surveying nonrespondents.” Field Methods 16(1): 23-54. DOI: https:// doi.org/10.1177/1525822X03259479.

Stoop, I., A. Koch, V. Halbherr, R. Fitzgerald, and S. Widdop. 2014. Field procedures in the European Social Survey Round 7: Enhancing response rates. The Hague: European Social Survey, SCP. Available at: http://www.europeansocialsurvey.org (accessed June 2020).

Tarnai, J. and D.L. Moore. 2008. "Measuring and improving telephone interviewer performance and productivity." In Advances in telephone survey methodology, edited by E. de Leeuw, L. Japec, P.J. Lavrakas, M.W. Link, and R.L. Sangster. pp. 359-384, Hoboken, New Jersey: John Wiley \& Sons.

West, B.T. and K. Olson. 2010. "How much of interviewer variance is really nonresponse error variance?” Public Opinion Quarterly 74(5): 1004-1026. DOI: https://doi.org/ $10.1093 / \mathrm{poq} / \mathrm{nfq} 061$.

Wright, G. 2015. “An empirical examination of the relationship between nonresponse rate and nonresponse bias." Statistical Journal of the IAOS 31(2): 305-315. DOI: https:// doi.org/10.3233/sji-140844.

Wuyts, C., L. Jacobs, D. de Coninck, P. Italiano, and G. Loosveldt. 2018. European Social Survey Round 8 Belgium: Process evaluation for data collection. Leuven: KU Leuven.

Received September 2018

Revised April 2019

Accepted April 2020 